\title{
Quantization of Systems with Constraints*
}

\author{
John R. Klauder \\ Departments of Physics and Mathematics \\ University of Florida \\ Gainesville, Fl 32611
}

\begin{abstract}
New measures for the quantization of systems with constraints are discussed and applied to several examples, in particular, examples of alternative but equivalent formulations of given first-class constraints, as well as a comparison of both regular and irregular constraints.
\end{abstract}

\section{Introduction}

The quantization of systems with constraints is of considerable importance in a variety of applications. Let $\left\{p_{j}, q^{j}\right\}, 1 \leq j \leq J$, denote a set of dynamical variables, $\left\{\lambda^{a}\right\}, 1 \leq a \leq A \leq 2 J$, a set of Lagrange multipliers, and $\left\{\phi_{a}(p, q)\right\}$ a set of constraints. Then the dynamics of a constrained system may be summarized in the form of an action principle by means of the classical action (summation implied)

$$
I=\int\left[p_{j} \dot{q}^{j}-H(p, q)-\lambda^{a} \phi_{a}(p, q)\right] d t .
$$

The resultant equations of motion that arise from the action read

$$
\dot{q}^{j}=\frac{\partial H(p, q)}{\partial p_{j}}+\lambda^{a} \frac{\partial \phi_{a}(p, q)}{\partial p_{j}} \equiv\left\{q^{j}, H\right\}+\lambda^{a}\left\{q^{j}, \phi_{a}\right\},
$$

*Based on a presentation at the International Symposium Symmetries in Science IX, Bregenz, Austria, August 6-10, 1996. 


$$
\begin{aligned}
& \dot{p}_{j}=-\frac{\partial H(p, q)}{\partial q^{j}}-\lambda^{a} \frac{\partial \phi_{a}(p, q)}{\partial q^{j}} \equiv\left\{p_{j}, H\right\}+\lambda^{a}\left\{p_{j}, \phi_{a}\right\} \\
& \phi_{a}(p, q)=0
\end{aligned}
$$

where $\{\cdot, \cdot\}$ denotes the Poisson bracket. The set of conditions $\left\{\phi_{a}(p, q)=0\right\}$ define the constraint hypersurface. If the constraints satisfy

$$
\begin{aligned}
& \left\{\phi_{a}(p, q), \phi_{b}(p, q)\right\}=c_{a b}{ }^{c} \phi_{c}(p, q), \\
& \left\{\phi_{a}(p, q), H(p, q)\right\}=h_{a}{ }^{b} \phi_{b}(p, q),
\end{aligned}
$$

then we are dealing with a system of first-class constraints. If the coefficients $c_{a b}{ }^{c}$ and $h_{a}{ }^{b}$ are constants, then it is a system of closed first-class constraints; if they are suitable functions of the variables $p$ and $q$, then it is a system of open first-class constraints. If the first or both of the conditions in (3) and (4) fail, then the system involves second-class constraints.

For first-class constraints it is sufficient to impose the constraints at the initial time inasmuch as the equations of motion will ensure that the constraints are fulfilled at all future times. Such an initial imposition of the constraints is called an initial value equation. Furthermore, the Lagrange multipliers are not determined by the equations of motion; rather they must be specified (a choice of "gauge") in order for a solution of the dynamical equations to be given. For second-class constraints, on the other hand, the Lagrange multipliers are determined by the equations of motion in such a way that the constraints are satisfied for all time.

In the remainder of this section we briefly review standard quantization procedures for systems with closed first-class constraints, both of the operator and path integral variety, pointing out some problems along the way. In the following section we develop our coherent state approach for closed first-class constraints, which are illustrated by examples in the final section. For further details of such general systems, as well as a discussion of open first-class and second-class constraints, see Ref. [1].

\section{Standard operator quantization}

For a system of closed first-class constraints we assume (with $\hbar=1$ ) that

$$
\begin{aligned}
& {\left[\Phi_{a}(P, Q), \Phi_{b}(P, Q)\right]=i c_{a b}{ }^{c} \Phi_{c}(P, Q),} \\
& {\left[\Phi_{a}(P, Q), \mathcal{H}(P, Q)\right]=i h_{a}^{b} \Phi_{b}(P, Q),}
\end{aligned}
$$


where $\Phi_{a}$ and $\mathcal{H}$ denote self-adjoint constraint and Hamiltonian operators, respectively. Following Dirac [2], we adopt the quantization prescription given by

$$
i \dot{W}(P, Q)=[W(P, Q), \mathcal{H}(P, Q)]
$$

where $W$ denotes any function of the kinematical operators $\left\{Q^{j}\right\}$ and $\left\{P_{j}\right\}$ which are taken as a self-adjoint, irreducible representation of the commutation rules $\left[Q^{j}, P_{k}\right]=i \delta_{k}^{j} 1$ with all other commutators vanishing. The equations of motion hold for all time $t$, say $0<t<T$. On the other hand, the conditions

$$
\Phi_{a}(P, Q)|\psi\rangle_{\mathrm{phys}}=0
$$

that determine the physical Hilbert space are imposed only at time $t=0$ as the analog of the initial value equation; the quantum equations of motion ensure that the constraint conditions are fulfilled for all time.

The procedure of Dirac has potential difficulties if zero lies in the continuous spectrum of the constraint operators for in that case there are no normalizable solutions of the constraint condition (8). We face the same problem, of course, and our resolution is discussed below; see also Ref. [1].

\section{Standard path integral quantization}

Faddeev [3] has given a path integral formulation in the case of closed firstclass constraint systems as follows. The formal path integral

$$
\begin{aligned}
& \int \exp \left\{i \int_{0}^{T}\left[p_{j} \dot{q}^{j}-H(p, q)-\lambda^{a} \phi_{a}(p, q)\right] d t\right\} \mathcal{D} p \mathcal{D} q \mathcal{D} \lambda \\
& =\int \exp \left\{i \int_{0}^{T}\left[p_{j} \dot{q}^{j}-H(p, q)\right] d t\right\} \delta\{\phi(p, q)\} \mathcal{D} p \mathcal{D} q
\end{aligned}
$$

may well encounter divergences in the remaining integrals. Therefore, subsidiary conditions in the form $\chi^{a}(p, q)=0,1 \leq a \leq A$, are imposed picking out (ideally) one gauge equivalent point per gauge orbit, and in addition a factor (in the form of a determinant) is introduced to formally preserve canonical covariance. The result is the path integral

$$
\int \exp \left\{i \int_{0}^{T}\left[p_{j} \dot{q}^{j}-H(p, q)\right] d t\right\} \delta\{\chi(p, q)\} \operatorname{det}\left(\left\{\chi^{a}, \phi_{b}\right\}\right) \delta\{\phi(p, q)\} \mathcal{D} p \mathcal{D} q
$$


This result may also be expressed as

$$
\int \exp \left\{i \int_{0}^{T}\left[p_{j}^{*} \dot{q}^{* j}-H^{*}\left(p^{*}, q^{*}\right)\right] d t\right\} \mathcal{D} p^{*} \mathcal{D} q^{*},
$$

namely, as a path integral over a reduced phase space in which the $\delta$ functionals have been used to eliminate $2 A$ integration variables.

The final expression generally involves curvilinear phase-space coordinates for which the definition of the path integral is typically ill defined. Additionally, in the form (10), the Faddeev-Popov determinant often suffers from ambiguities connected with inadmissible gauge fixing conditions [ [ 1 ]. Thus this widely used prescription is not without its difficulties.

\section{BRST-BFV formulation}

By extending the phase space to include Grassmann variables, it is possible to develop alternative and more powerful methods to discuss systems with constraints. These methods are well documented, e.g., [5], and will not be discussed here. Instead, our interest focuses on what can be said without enlarging the number of variables beyond those that make up the original phase space augmented by the necessary Lagrange multipliers.

\section{Coherent State Path Integral}

Canonical coherent states may be defined by the relation

$$
|p, q\rangle \equiv e^{-i q^{j} P_{j}} e^{i p_{j} Q^{j}}|0\rangle
$$

where $|0\rangle$ traditionally denotes a normalized, unit frequency, harmonic oscillator ground state. Here $\left\{Q^{j}\right\}$ and $\left\{P_{j}\right\}, 1 \leq j \leq J$, denote an irreducible set of self-adjoint operators satisfying the Heisenberg commutation relations. The coherent states admit a resolution of unity in the form

$$
1=\int|p, q\rangle\langle p, q| d \mu(p, q), \quad d \mu(p, q) \equiv d^{J} p d^{J} q /(2 \pi)^{J},
$$

where the integration is over $\mathbb{R}^{2 J}$ and this integration domain and the form

of the measure are unique. For a general operator $\mathcal{H}(P, Q)$ we introduce the upper symbol

$$
H(p, q) \equiv\langle p, q|\mathcal{H}(P, Q)| p, q\rangle=\langle p, q|: H(P, Q):| p, q\rangle
$$


which is related to the normal-ordered form as shown. If $\mathcal{H}$ denotes the quantum Hamiltonian, then we shall adopt $H(p, q)$ as the classical Hamiltonian. We note that in the general case $H(p, q) \neq \mathcal{H}(p, q)$ but differ by terms which are $O(\hbar)$. Although these functions may be numerically different, in most cases the difference between these two functions is qualitatively insignificant. As remarked below, however, there are analogous cases where the qualitative difference is quite significant. Lastly, we also note that an important one-form is given by $i\langle p, q|d| p, q\rangle=p_{j} d q^{j}$.

Using these quantities, the coherent state path integral for the timedependent Hamiltonian $\mathcal{H}(P, Q)+\lambda^{a}(t) \Phi_{a}(P, Q)$ is readily given by

$$
\begin{aligned}
\left\langle p^{\prime \prime},\right. & \left.q^{\prime \prime}\left|\mathrm{T} e^{-i \int_{0}^{T}\left[\mathcal{H}(P, Q)+\lambda^{a}(t) \Phi_{a}(P, Q)\right] d t}\right| p^{\prime}, q^{\prime}\right\rangle \\
& =\lim _{\epsilon \rightarrow 0} \int \prod_{l=0}^{N}\left\langle p_{l+1}, q_{l+1}\left|e^{-i \epsilon\left(\mathcal{H}+\lambda_{l}^{a} \Phi_{a}\right)}\right| p_{l}, q_{l}\right\rangle \prod_{l=1}^{N} d \mu\left(p_{l}, q_{l}\right) \\
& =\int \exp \left\{i \int\left[i\langle p, q|(d / d t)| p, q\rangle-\left\langle p, q\left|\mathcal{H}+\lambda^{a} \Phi_{a}\right| p, q\right\rangle\right] d t\right\} \mathcal{D} \mu(p, q) \\
& =\mathcal{M} \int \exp \left\{i \int\left[p_{j} \dot{q}^{j}-H(p, q)-\lambda^{a} \phi_{a}(p, q)\right] d t\right\} \mathcal{D} p \mathcal{D} q
\end{aligned}
$$

In the second line we have set $p_{N+1}, q_{N+1}=p^{\prime \prime}, q^{\prime \prime}$ and $p_{0}, q_{0}=p^{\prime}, q^{\prime}$, and repeatedly inserted the resolution of unity; in the third and fourth lines we have formally interchanged the continuum limit and the integrations, and written for the integrand the form it assumes for continuous and differential paths $(\mathcal{M}$ denotes a formal normalization constant). The result evidently depends on the chosen form of the functions $\left\{\lambda^{a}(t)\right\}$.

\section{Enforcing the quantum constraints}

Let us next introduce the quantum analog of the initial value equation. For simplicity we assume that the constraint operators generate a compact group; the case of a noncompact group is implicitly discussed below (see Ref. [1]). In that case

$$
\mathbb{E} \equiv \int e^{-i \xi^{a} \Phi_{a}(P, Q)} \delta \xi
$$

defines a projection operator onto the subspace for which $\Phi_{a}=0$ provided that $\delta \xi$ denotes the normalized, $\int \delta \xi=1$, group invariant measure. Based on 
(5), (6), and (16) it follows that

$$
\begin{gathered}
e^{-i \tau^{a} \Phi_{a}} \mathbb{E}=\mathbb{E}, \\
e^{-i \mathcal{H} T} \mathbb{E}=\mathbb{E} e^{-i \mathcal{H} T} \mathbb{E}=\mathbb{E} e^{-i(\mathbf{E} \mathcal{H} \mathbf{E}) T} \mathbb{E}
\end{gathered}
$$

We now project the propagator (15) onto the quantum constraint subspace which leads to the following set of relations

$$
\begin{aligned}
\int\left\langle p^{\prime \prime},\right. & \left.q^{\prime \prime}\left|\mathrm{T} e^{-i \int\left[\mathcal{H}+\lambda^{a}(t) \Phi_{a}\right] d t}\right| \bar{p}^{\prime}, \bar{q}^{\prime}\right\rangle\left\langle\bar{p}^{\prime}, \bar{q}^{\prime}|\mathbb{E}| p^{\prime}, q^{\prime}\right\rangle d \mu\left(\bar{p}^{\prime}, \bar{q}^{\prime}\right) \\
& =\left\langle p^{\prime \prime}, q^{\prime \prime}\left|\mathrm{T} e^{-i \int\left[\mathcal{H}+\lambda^{a}(t) \Phi_{a}\right] d t} \mathbb{E}\right| p^{\prime}, q^{\prime}\right\rangle \\
& =\lim \left\langle p^{\prime \prime}, q^{\prime \prime}\left|\left[\prod_{l}^{\overleftarrow{l}}\left(e^{-i \epsilon \mathcal{H}} e^{-i \epsilon \lambda_{l}^{a} \Phi_{a}}\right)\right] \mathbb{E}\right| p^{\prime}, q^{\prime}\right\rangle \\
& =\left\langle p^{\prime \prime}, q^{\prime \prime}\left|e^{-i T \mathcal{H}} e^{-i \tau^{a} \Phi_{a}} \mathbb{E}\right| p^{\prime}, q^{\prime}\right\rangle \\
& =\left\langle p^{\prime \prime}, q^{\prime \prime}\left|e^{-i T \mathcal{H}} \mathbb{E}\right| p^{\prime}, q^{\prime}\right\rangle
\end{aligned}
$$

where $\tau^{a}$ incorporates the functions $\lambda^{a}$ as well as the structure parameters $c_{a b}{ }^{c}$ and $h_{a}^{b}$. Alternatively, this expression has the formal path integral representation

$$
\int \exp \left\{i \int\left[p_{j} \dot{q}^{j}-H(p, q)-\lambda^{a} \phi_{a}(p, q)\right] d t-i \xi^{a} \phi_{a}(p, q)\right\} \mathcal{D} \mu(p, q) \delta \xi .
$$

On comparing (19) and (20) we observe that after projection onto the quantum constraint subspace the propagator is entirely independent of the choice of the Lagrange multiplier functions. In other words, the projected propagator is gauge invariant; see Refs. [1, 5, 6].

We may also express the physical (projected) propagator in a more general form, namely,

$$
\begin{gathered}
\int \exp \left\{i \int\left[p_{j} \dot{q}^{j}-H(p, q)-\lambda^{a} \phi_{a}(p, q)\right] d t\right\} \mathcal{D} \mu(p, q) \mathcal{D} C(\lambda) \\
=\left\langle p^{\prime \prime}, q^{\prime \prime}\left|e^{-i T \mathcal{H}} \mathbb{E}\right| p^{\prime}, q^{\prime}\right\rangle
\end{gathered}
$$

provided that $\int \mathcal{D} C(\lambda)=1$ and that such an average over the functions $\left\{\lambda^{a}\right\}$ introduces (at least) one factor $\mathbb{E}$. 


\section{Reproducing kernel Hilbert spaces}

The coherent state matrix elements of $\mathbb{E}$ define a fundamental kernel

$$
\mathcal{K}\left(p^{\prime \prime}, q^{\prime \prime} ; p^{\prime}, q^{\prime}\right) \equiv\left\langle p^{\prime \prime}, q^{\prime \prime}|\mathbb{E}| p^{\prime}, q^{\prime}\right\rangle,
$$

which is a bounded, continuous function for any projection operator $\mathbb{E}$, especially including the unit operator. It follows that $\mathcal{K}\left(p^{\prime \prime}, q^{\prime \prime} ; p^{\prime}, q^{\prime}\right)^{*}=$ $\mathcal{K}\left(p^{\prime}, q^{\prime} ; p^{\prime \prime}, q^{\prime \prime}\right)$ as well as

$$
\sum_{k, l=1}^{K} \alpha_{k}^{*} \alpha_{l} \mathcal{K}\left(p_{k}, q_{k} ; p_{l}, q_{l}\right) \geq 0
$$

for all sets $\left\{\alpha_{k}\right\},\left\{p_{k}, q_{k}\right\}$, and all $K<\infty$. The last relation is an automatic consequence of the complex conjugate property and the fact that

$$
\mathcal{K}\left(p^{\prime \prime}, q^{\prime \prime} ; p^{\prime}, q^{\prime}\right)=\int \mathcal{K}\left(p^{\prime \prime}, q^{\prime \prime} ; p, q\right) \mathcal{K}\left(p, q ; p^{\prime}, q^{\prime}\right) d \mu(p, q)
$$

holds in virtue of the coherent state resolution of unity and the properties of $\mathbb{E}$. The function $\mathcal{K}$ is called the reproducing kernel and the Hilbert space it engenders is termed a reproducing kernel Hilbert space. A dense set of elements in the Hilbert space is given by functions of the form

$$
\psi(p, q)=\sum_{k=1}^{K} \alpha_{k} \mathcal{K}\left(p, q ; p_{k}, q_{k}\right),
$$

and the inner product of this function with itself has two equivalent forms given by

$$
\begin{aligned}
(\psi, \psi) & =\sum_{k, l=1}^{K} \alpha_{k}^{*} \alpha_{l} \mathcal{K}\left(p_{k}, q_{k} ; p_{l}, q_{l}\right) \\
& =\int \psi(p, q)^{*} \psi(p, q) d \mu(p, q) .
\end{aligned}
$$

As usual, the inner product of two distinct functions may be determined by polarization. Clearly the entire Hilbert space is characterized by the reproducing kernel $\mathcal{K}$. Change the kernel $\mathcal{K}$ and one changes the representation of the Hilbert space. Following a suitable limit, it is even possible to change the dimension of the Hilbert space, as we discuss in the next section. 


\section{Reduction of the reproducing kernel}

Suppose the reproducing kernel depends on a number of variables and additional parameters. We can generate new reproducing kernels from a given one by a variety of means. For example, the expressions

$$
\begin{aligned}
& \mathcal{K}_{1}\left(p^{\prime \prime} ; p^{\prime}\right)=\mathcal{K}\left(p^{\prime \prime}, c ; p^{\prime}, c\right), \\
& \mathcal{K}_{2}\left(p^{\prime \prime} ; p^{\prime}\right)=\int w\left(q^{\prime \prime}\right)^{*} w\left(q^{\prime}\right) \mathcal{K}\left(p^{\prime \prime}, q^{\prime \prime} ; p^{\prime}, q^{\prime}\right) d q^{\prime \prime} d q^{\prime}, \\
& \mathcal{K}_{3}\left(p^{\prime \prime}, q^{\prime \prime} ; p^{\prime}, q^{\prime}\right)=\lim \mathcal{K}\left(p^{\prime \prime}, q^{\prime \prime} ; p^{\prime}, q^{\prime}\right)
\end{aligned}
$$

each generate a new reproducing kernel provided the resultant function remains continuous. Sometimes, however, the inner product in the Hilbert space generated by the new reproducing kernel is only given by an analog of (26) and not by (27), although frequently some sort of local integral representation for the inner product may also exist.

Let us present an example of the reduction of a reproducing kernel. Consider the example

$$
\begin{aligned}
& \left\langle p^{\prime \prime}, q^{\prime \prime}|\mathbb{E}| p^{\prime}, q^{\prime}\right\rangle \\
& \quad=\pi^{-1 / 2} \int_{-\delta}^{\delta} \exp \left[-\frac{1}{2}\left(k-p^{\prime \prime}\right)^{2}+i k\left(q^{\prime \prime}-q^{\prime}\right)-\frac{1}{2}\left(k-p^{\prime}\right)^{2}\right] d k,
\end{aligned}
$$

which defines a reproducing kernel for any $\delta>0$ that corresponds to an infinite dimensional Hilbert space. Let us multiply this expression by $\pi^{1 / 2} /(2 \delta)$ and take the limit $\delta \rightarrow 0$. The result is the expression

$$
\mathcal{K}\left(p^{\prime \prime} ; p^{\prime}\right)=e^{-\frac{1}{2}\left(p^{\prime \prime 2}+p^{\prime 2}\right)},
$$

which has become a reproducing kernel that characterizes a one-dimensional Hilbert space with every functional representative proportional to $\chi(p) \equiv$ $\exp \left(-p^{2} / 2\right)$. This one-dimensional Hilbert space representation also admits a local integral representation for the inner product given by

$$
(\chi, \chi)=\int|\chi(p)|^{2} d p / \sqrt{\pi} .
$$

This example is an important one inasmuch as it shows how a constraint operator with a continuous spectrum is dealt with in the coherent state approach. 


\section{Applications}

\section{Example 1}

The following example is based on Problem 5.1 in Ref. [5]. Consider the two-degree of freedom system with vanishing Hamiltonian described by the classical action

$$
I=\int\left(p_{1} \dot{q}_{1}+p_{2} \dot{q}_{2}-\lambda_{1} p_{1}-\lambda_{2} p_{2}\right) d t
$$

For notational convenience all indices have been placed as subscripts. The equations of motion become

$$
\dot{q}_{j}=\lambda_{j}, \quad \dot{p}_{j}=0, \quad p_{j}=0, \quad j=1,2 \text {. }
$$

Evidently the Poisson bracket $\left\{p_{1}, p_{2}\right\}=0$.

As a second version of the same dynamics, consider the classical action

$$
I=\int\left(p_{1} \dot{q}_{1}+p_{2} \dot{q}_{2}-\lambda_{1} p_{1}-\lambda_{2} e^{c q_{1}} p_{2}\right) d t
$$

with $c$ a constant, which leads to the equations of motion

$$
\dot{q}_{1}=\lambda_{1}, \quad \dot{q}_{2}=\lambda_{2} e^{c q_{1}}, \quad \dot{p}_{1}=-c \lambda_{2} e^{c q_{1}} p_{2}, \quad \dot{p}_{2}=0, \quad p_{1}=e^{c q_{1}} p_{2}=0 .
$$

Since $e^{c q_{1}} p_{2}=0$ implies that $p_{2}=0$, it follows that the two formulations are equivalent despite the fact that in the second case $\left\{p_{1}, e^{c q_{1}} p_{2}\right\}=-c e^{c q_{1}} p_{2}$, which has a completely different algebraic structure when $c \neq 0$ as compared to $c=0$.

Let us discuss these two examples from the point of view of a coherent state quantization. For the first version we consider

$$
\mathcal{M} \int \exp \left[i \int\left(p_{1} \dot{q}_{1}+p_{2} \dot{q}_{2}-\lambda_{1} p_{1}-\lambda_{2} p_{2}\right) d t\right] \mathcal{D} p \mathcal{D} q \mathcal{D} C(\lambda)
$$

which is defined in a fashion to yield

$$
\left\langle p^{\prime \prime}, q^{\prime \prime}|\mathbb{E}| p^{\prime}, q^{\prime}\right\rangle
$$

where

$$
\mathbb{E}=\mathbb{E}\left(-\delta<P_{1}<\delta\right) \mathbb{E}\left(-\delta<P_{2}<\delta\right)
$$


In particular this leads to the fact that

$$
\begin{gathered}
\left\langle p^{\prime \prime}, q^{\prime \prime}|\mathbb{E}| p^{\prime}, q^{\prime}\right\rangle \\
=\pi^{-1} \prod_{l=1}^{2} \int_{-\delta}^{\delta} \exp \left[-\frac{1}{2}\left(k_{l}-p_{l}^{\prime \prime}\right)^{2}+i k_{l}\left(q_{l}^{\prime \prime}-q_{l}^{\prime}\right)-\frac{1}{2}\left(k_{l}-p_{l}^{\prime}\right)^{2}\right] d k_{l} .
\end{gathered}
$$

Let us reduce this reproducing kernel by multiplying this expression by $\pi /(2 \delta)^{2}$ and passing to the limit $\delta \rightarrow 0$. The result is the reduced reproducing kernel given by

$$
\exp \left[-\frac{1}{2}\left(p_{1}^{\prime \prime 2}+p_{2}^{\prime \prime 2}\right)\right] \exp \left[-\frac{1}{2}\left(p_{1}^{\prime 2}+p_{2}^{\prime 2}\right)\right],
$$

which clearly characterizes a particular representation of a one-dimensional Hilbert space in which every vector is proportional to $\exp \left[-\frac{1}{2}\left(p_{1}^{2}+p_{2}^{2}\right)\right]$. This example is, of course, related to the reduction example given earlier. Moreover, we can introduce a local integral representation over the remaining $p$ variables for the inner product if we so desire.

Let us now turn attention to the second formulation of the problem by focusing (for a different $C(\lambda)$ ) on

$$
\mathcal{M} \int \exp \left[i \int\left(p_{1} \dot{q}_{1}+p_{2} \dot{q}_{2}-\lambda_{1} p_{1}-\lambda_{2} e^{c q_{1}} p_{2}\right) d t\right] \mathcal{D} p \mathcal{D} q \mathcal{D} C(\lambda)
$$

This expression again leads (for a different $\mathbb{E}$ ) to

$$
\left\langle p^{\prime \prime}, q^{\prime \prime}|\mathbb{E}| p^{\prime}, q^{\prime}\right\rangle
$$

where in the present case the fully reduced form of this expression is proportional to

$$
\begin{aligned}
& \int \exp [\left.-\frac{1}{2}\left(k_{2}-p_{2}^{\prime \prime}\right)^{2}+i k_{2}\left(q_{2}^{\prime \prime}-q_{2}^{\prime}\right)-\frac{1}{2}\left(k_{2}-p_{2}^{\prime}\right)^{2}\right] \\
& \times \exp \left[-\frac{1}{2}\left(k_{1}-p_{1}^{\prime \prime}\right)^{2}+i k_{1} q_{1}^{\prime \prime}-\frac{1}{2} i \lambda_{1} k_{1}\right] \\
& \times \exp \left[-i x k_{1}-i \lambda_{2} e^{c x} k_{2}+i x \kappa_{1}\right] \\
& \times \exp \left[-\frac{1}{2} i \lambda_{1} \kappa_{1}-i \kappa_{1} q_{1}^{\prime}-\frac{1}{2}\left(\kappa_{1}-p_{1}^{\prime}\right)^{2}\right] \\
& \times d k_{2} d k_{1} d x d \kappa_{1} d \lambda_{1} d \lambda_{2} .
\end{aligned}
$$

When normalized appropriately, this expression is evaluated as

$$
\exp \left[-\frac{1}{2}\left(p_{1}^{\prime \prime 2}+p_{2}^{\prime \prime 2}+i c p_{1}^{\prime \prime}\right)\right] \exp \left[-\frac{1}{2}\left(p_{1}^{\prime 2}+p_{2}^{\prime 2}-i c p_{1}^{\prime}\right)\right]
$$


which once again represents a one-dimensional Hilbert space.

Thus we have obtained a $c$-dependent family of distinct but equivalent quantum representations for the same Hilbert space, reflecting the $c$ dependent family of equivalent classical solutions. Observe that in the quantum theory, just as in the classical theory, all observable effects are independent of $c$.

\section{Example 2}

In discussing constraints one often pays considerable attention to the regularity of the expressions involved; see [5], Sec. 1.1.2. Consider, once again, the simple example of a single constraint $p=0$ as illustrated by the classical action

$$
I=\int(p \dot{q}-\lambda p) d t
$$

The equations of motion read $\dot{q}=\lambda, \dot{p}=0$, and $p=0$. On the other hand, one may ask about imposing the constraint $p^{3}=0$ or possibly $p^{1 / 3}=0$, etc., instead of $p=0$. Let us incorporate several such odd (function) examples by studying the classical action

$$
\int\left(p \dot{q}-\lambda p|p|^{\gamma}\right) d t, \quad \gamma>-1
$$

Here the equations of motion include $\dot{q}=\lambda(\gamma+1)|p|^{\gamma}$ which, along with the constraint $p|p|^{\gamma}=0$, may cause some difficulty in seeking a classical solution of the equations of motion, e.g., if $\gamma<0$. When $\gamma \neq 0$, such constraints are said to be irregular. It is clear from (9) that irregular constraints lead to considerable difficulty in conventional phase-space path integral approaches.

Let us examine the question of irregular constraints from the point of view of a coherent state, phase-space path integral quantization. For any

$\gamma>-1$, we first observe that the operator $P|P|^{\gamma}$ is well defined by means of its spectral decomposition. Moreover, it follows that

$$
\begin{aligned}
& \int e^{-i \xi P|P|^{\gamma}} \frac{\sin \left(\delta^{\gamma+1} \xi\right)}{\pi \xi} d \xi \\
& \quad=\mathbb{E}\left(-\delta^{\gamma+1}<P|P|^{\gamma}<\delta^{\gamma+1}\right) \\
& =\mathbb{E}(-\delta<P<\delta) .
\end{aligned}
$$


Thus, from the operator point of view, it is possible to consider the constraint operator $P|P|^{\gamma}$ just as easily as $P$ itself. In particular, it follows that

$$
\left\langle p^{\prime \prime}, q^{\prime \prime}|\mathbb{E}| p^{\prime}, q^{\prime}\right\rangle=\mathcal{M} \int \exp \left[i \int\left(p \dot{q}-\lambda p|p|^{\gamma}\right) d t\right] \mathcal{D} p \mathcal{D} q \mathcal{D} C_{\gamma}(\lambda)
$$

where we have appended $\gamma$ to the measure for the Lagrange multiplier $\lambda$ to emphasize the dependence of that measure on $\gamma$. The reduction of the reproducing kernel proceeds exactly like the cases discussed earlier, and we determine for all $\gamma>-1$ that

$$
\lim _{\delta \rightarrow 0} \frac{\sqrt{\pi}}{(2 \delta)}\left\langle p^{\prime \prime}, q^{\prime \prime}|\mathbb{E}| p^{\prime}, q^{\prime}\right\rangle=e^{-\frac{1}{2}\left(p^{\prime \prime 2}+p^{2}\right)},
$$

representative of a one-dimensional Hilbert space. Just like the classical theory, note that the ultimate form of the quantum theory is independent of $\gamma$.

It is natural to ask how one is to understand this acceptable behavior for the quantum theory for irregular constraints while there are difficulties that seem to be present in the classical theory. In the first section we discussed the definition of the classical generator as derived from the quantum generator. Just like the classical and quantum Hamiltonians, the connection between the classical and quantum constraints is given by

$$
\phi(p, q) \equiv\langle p, q|\Phi(P, Q)| p, q\rangle=\langle 0|\Phi(P+p, Q+q)| 0\rangle .
$$

With this rule we typically find that $\phi(p, q) \neq \Phi(p, q)$ due to the fact that $\hbar \neq 0$, but the difference between these expressions is generally qualitatively unimportant. In certain circumstances, however, that difference is qualitatively significant even though it is quantitatively very small. Since that difference is $O(\hbar)$ let us explicitly exhibit the appropriate $\hbar$-dependence hereafter. First consider the case of $\gamma=2$. In that case

$$
\left\langle p, q\left|P^{3}\right| p, q\right\rangle=\left\langle 0\left|(P+p)^{3}\right| 0\right\rangle=p^{3}+3\left\langle P^{2}\right\rangle p
$$

where we have introduced the shorthand $\langle(\cdot)\rangle \equiv\langle 0|(\cdot)| 0\rangle$. Since $\left\langle P^{2}\right\rangle=\hbar / 2$ it follows that for the quantum constraint $P^{3}$, the corresponding classical constraint function is given by $p^{3}+(3 \hbar / 2) p$. For $|p| \gg \sqrt{\hbar}$, this constraint is adequately given by $p^{3}$. However, when $|p| \ll \sqrt{\hbar}$ - as must eventually be the 
case in order to actually satisfy the classical constraint - then the functional form of the constraint is effectively $(3 \hbar / 2) p$. In short, if the quantum constraint operator is $P^{3}$, then the classical constraint function is in fact regular when the constraint vanishes.

A similar discussion holds for a general value of $\gamma$. The classical constraint is given by

$$
\begin{aligned}
\phi_{\gamma}(p) & =(\pi \hbar)^{-1 / 2} \int(k+p)|k+p|^{\gamma} e^{-k^{2} / \hbar} d k \\
& =(\pi \hbar)^{-1 / 2} \int k|k|^{\gamma} e^{-(k-p)^{2} / \hbar} d k
\end{aligned}
$$

For $|p| \gg \sqrt{\hbar}$ the first line of this expression effectively yields $\phi_{\gamma}(p) \simeq p|p|^{\gamma}$. On the other hand, for $p \approx 0$, and more especially for $|p| \ll \sqrt{\hbar}$, the second line of this expression shows that this constraint function vanishes linearly, speci fically as $\phi_{\gamma}(p) \simeq k p$, where

$$
k \equiv 2\left(\hbar^{\gamma} / \pi\right)^{1 / 2} \int y^{2}|y|^{\gamma} e^{-y^{2}} d y=2\left(\hbar^{\gamma} / \pi\right)^{1 / 2} \Gamma((\gamma+3) / 2) \equiv \hbar^{\gamma / 2} k_{o} .
$$

A rough, but qualitatively correct expression for this behavior is given by

$$
\phi_{\gamma}(p) \simeq k_{o} p\left(\hbar+p^{2} k_{o}^{-2 / \gamma}\right)^{\gamma / 2}
$$

Thus, from the present point of view, irregular constraints do not arise from consistent quantum constraints; instead, irregular constraints arise as limiting expressions of certain consistent, regular classical constraints as $\hbar \rightarrow$ 0 .

There is one category of irregular constraints that is not covered by the foregoing discussion, namely the even constraints, e.g., a classical constraint given by $p^{2}=0$ rather than $p=0$. This case differs from those treated above because the classical constraint is strictly nonnegative. However, from the operator point of view, the case of $P^{2}$ is not qualitatively different from the other cases because we still have

$$
\begin{aligned}
\int e^{-i \xi P^{2}} \frac{\sin \left(\delta^{2} \xi\right)}{\pi \xi} d \xi \\
=\mathbb{E}\left(P^{2}<\delta^{2}\right) \\
=\mathbb{E}(-\delta<P<\delta) .
\end{aligned}
$$


From the classical point of view, however, it follows that

$$
\phi(p)=p^{2}+\left\langle P^{2}\right\rangle=p^{2}+\frac{1}{2} \hbar,
$$

which evidently never vanishes so long as $\hbar>0$. This result is not surprising; as the expectation value of a nonnegative operator with continuous spectrum, it cannot vanish. If we adopt our previous interpretation that there are no irregular odd constraints thanks to a nonvanishing $\hbar$, then we are forced to admit that for even constraints there is no suitable classical analog. Of course, such examples are in no way restricted to terms involving $\hbar$. One need only consider the classical constraint $p^{2}+q^{2}+1=0$ which evidently has no solution for real phase space variables.

However, there is another way to look at even classical constraints [7]. For the sake of illustration, let us initially focus on the simple case $p^{2}=0$ as an even representative of the odd classical constraint $p=0$. From a simple physical point of view, a system will follow a constraint at least approximately provided it costs a great deal of energy to violate it. onsider the simple system described by the action functional

$$
I=\int\left(p \dot{q}-\frac{1}{2} A p^{2}\right) d t
$$

where $A$ is a large positive constant. The equations of motion for this case read $\dot{q}=A p$, and $\dot{p}=0$, and the solution to these equations of motion is $p(t)=p^{\prime}$ and $q(t)=A p^{\prime} t+q^{\prime}$. We insist that the solution should be independent of the large parameter $A$, and so it follows that $p^{\prime}=O\left(A^{-1}\right)$. As a consequence, the "energy" $A p^{2} / 2=O\left(A^{-1}\right)$ as well. In the limit that $A \rightarrow$ $\infty$, it follows that $p^{\prime}=0$, the "energy" vanishes, while $q(t)=c t+q^{\prime}$ for some $c$. This solution is seen to be an example of one from the usual formulation using a Lagrange multiplier. Indeed, we can also make $q(t)$ into a rather general function of time by allowing for $A$ to be time dependent as well as large; however, for convenience, we ignore this rather evident generalization. An alternative example of the same kind is given by

$$
I=\int\left[p \dot{q}-\frac{1}{2} A\left(p^{2}+q^{2}-1\right)^{2}\right] d t .
$$

The analysis in this case proceeds as before with the final result that

$$
q(t)=q^{\prime} \cos (c t)+p^{\prime} \sin (c t) . \quad p(t)=-q^{\prime} \sin (c t)+p^{\prime} \cos (c t),
$$


subject to the requirement that $p^{2}+q^{\prime 2}=1$. Note well, in these cases, that we deal with even constraints and a large positive parameter $A$ in order that no cancellation among large terms of opposite sign is possible.

Following this brief introduction to an alternative classical formulation of a theory with a constraint, let us turn our attention to the quantum mechanics of such a system. Let $W$ denote the basic constraint operator we wish to maintain; for instance, $W=P^{2} / 2$ for the first case and $W=$ $\left(: P^{2}+Q^{2}:-1\right)^{2} / 2$ for the second case. Maintaining $\hbar=1$ temporarily, interest centers on the expression

$$
\lim K_{A} e^{-i T A W}
$$

as $A \rightarrow \infty$, where $K_{A}$ is a suitable $A$-dependent parameter. If $W=0$ is a point in the discrete spectrum, as in the second example, then $K_{A}=1$ and the result is a projection operator $\mathbb{E}$ onto the subspace where $W=0$. The case of a discrete spectrum is not especially difficult and we choose to focus our attention on the first example for which $W=0$ is a point in the continuous spectrum. In that case it follows that

$$
\begin{aligned}
& \lim _{A \rightarrow \infty} \sqrt{i A T / 2 \pi}\left\langle p^{\prime \prime}, q^{\prime \prime}\left|e^{-i T A P^{2} / 2}\right| p^{\prime}, q^{\prime}\right\rangle \\
&= \lim _{A \rightarrow \infty} \sqrt{i A T / 2 \pi} \int \exp \left[-\frac{1}{2}\left(k-p^{\prime \prime}\right)^{2}\right. \\
&\left.\quad+i k\left(q^{\prime \prime}-q^{\prime}\right)-i \frac{1}{2} T A k^{2}-\frac{1}{2}\left(k-p^{\prime}\right)^{2}\right] d k \\
&=\exp \left[-\frac{1}{2}\left(p^{\prime \prime 2}+p^{\prime 2}\right)\right],
\end{aligned}
$$

which is exactly the same result that we would find had we started with the usual formulation with a Lagrange multiplier.

Let us now present a coherent state path integral for this version of doing constraint systems. In particular we observe that

$$
\left\langle p^{\prime \prime}, q^{\prime \prime}\left|e^{-i T A P^{2} / 2}\right| p^{\prime}, q^{\prime}\right\rangle=\mathcal{M} \int \exp \left\{i \int\left[p \dot{q}-\frac{1}{2} A\left(p^{2}+\left\langle P^{2}\right\rangle\right)\right] d t\right\} \mathcal{D} p \mathcal{D} q \text {. }
$$

In this formal path integral we have included the term

$$
\left\langle p, q\left|P^{2}\right| p, q\right\rangle=p^{2}+\left\langle P^{2}\right\rangle=p^{2}+\frac{1}{2} \hbar \text {. }
$$

Thus, from the point of view of deriving the classical action from the quantum theory, we are not led to the original starting action (59) but instead to

$$
I=\int\left[p \dot{q}-\frac{1}{2} A\left(p^{2}+\frac{1}{2} \hbar\right)\right] d t .
$$


When a term such as (65) arose as the coefficient of a Lagrange multiplier, we concluded that it was unacceptable because it was impossible to satisfy $p^{2}+\hbar / 2=0$ as a constraint for real phase space variables. On the other hand, we now see such a term arise as a coefficient of the large parameter $A$. Note that the extra $\hbar$ term does not influence the equations of motion. In particular, the classical equations of motion are $\dot{q}=A p$ and $\dot{p}=0$ exactly as before. Thus the criterion that the classical solutions should be independent of the large parameter $A$ leads once again to the solutions $p(t)=0$ and $q(t)=c t+q^{\prime}$. It is true that the "energy" given by $A\left(p^{\prime 2}+\hbar / 2\right) / 2=A \hbar / 4$ diverges as $A \rightarrow \infty$, but that expression is not the real energy for any physical system. Thus we conclude that the appearance of the extra term in the classical action does not interfere with an appropriate solution of the classical equations of motion unlike the formulation with a Lagrange multiplier.

Let us briefly raise the issue in the case of another even constraint, say $p^{4}=0$ based on the classical action

$$
I=\int\left[p \dot{q}-\frac{1}{4} A p^{4}\right] d t .
$$

The equations of motion, $\dot{q}=A p^{3}$ and $\dot{p}=0$, lead to an $A$-independent form of the classical solution given by $q(t)=c t+q^{\prime}$, for some $c$, and $p(t)=0$. Quantum mechanically, we are led to conclude that

$$
\lim _{A \rightarrow \infty} k A^{1 / 4}\left\langle p^{\prime \prime}, q^{\prime \prime}\left|e^{-i T A P^{4} / 4}\right| p^{\prime}, q^{\prime}\right\rangle=\exp \left[-\frac{1}{2}\left(p^{\prime \prime 2}+p^{2}\right)\right],
$$

for a suitable constant $k$. The coherent state path integral formulation leads to

$$
\mathcal{M} \int \exp \left\{i \int\left[p \dot{q}-\frac{1}{4} A\left(p^{4}+6\left\langle P^{2}\right\rangle p^{2}+\left\langle P^{4}\right\rangle\right)\right] d t\right\} \mathcal{D} p \mathcal{D} q
$$

which implies that the proper factor in the classical theory is

$$
\frac{1}{4} A\left[p^{4}+3 \hbar p^{2}+(3 / 4) \hbar^{2}\right] .
$$

If we take this form seriously, we are led to conclude that as $A \rightarrow \infty$ and $|p| \ll \sqrt{\hbar}$, only the quadratic term becomes important in the equations of motion as compared with the quartic term. In short, the $P^{4}$ term is just as "regular" as the $P^{2}$ term. Thus, once again, we conclude that from the coherent state point of view there are no irregular constraints so long as $\hbar>0$; it is only in the limit where $\hbar \rightarrow 0$ that certain regular constraints turn into irregular constraints. 


\section{Acknowledgements}

Thanks are expressed to Bruno Gruber for his efforts which resulted in a very pleasant conference. Communications with J. Govaerts, M. Henneaux, and S. Shabanov, and discussions with B. Whiting are gratefully acknowledged.

\section{References}

[1] J.R. Klauder, "Coherent State Quantization of Constraint Systems", IHES/P/96/29, quant-ph/9604033, Annals of Physics (in press); see also quant-ph/9607019 and quant-ph/9607020.

[2] P.A.M. Dirac, Lectures on Quantum Mechanics (Belfer Graduate School of Science, Yeshiva University, New York, 1964).

[3] L.D. Faddeev, Theor. Math. Phys. 1, 1 (1970).

[4] V.N. Gribov, Nucl. Phys. B139, 1 (1978); I.M. Singer, Commun. Math. Phys. 60, 7 (1978); see also J. Govaerts, Hamiltonian Quantisation and Constrained Dynamics, Leuven Notes in Mathematical and Theoretical Physics, Vol. 4, Series B: Theoretical Particle Physics (Leuven University Press, 1991).

[5] M. Henneaux and C. Teitelboim, Quantization of Gauge Systems (Princeton University Press, 1992).

[6] S.V. Shabanov, JINR Lecture Notes, Volume 54, "Phase Space Structure in Gauge Theories", Dubna (1989) (in Russian); L.V. Prokhorov and S.V. Shabanov, Sov. Phys. Usp. 34, 108 (1991).

[7] V.I. Arnold, Mathematical Methods of Classical Mechanics (Springer Verlag, New York, 1978), Chap. 4. 